\title{
Coupled Multi-Disciplinary Simulation of Composite Engine Structures in Propulsion Environment
}

Christos C. Chamis

National Aeronautics and Space Administration

Lewis Research Center

Cleveland, Ohio

and

Surendra N. Singhal

Sverdrup Technology, Inc.

Lewis Research Center Group

Brook Park, Ohio

Prepared for the

International Gas Turbine Aeroengine Congress and Exposition Cologne, Germany, June 1-4, 1992

\section{N/Sก}

(NASA-IM-105575) COUPLFD MULTI-DISCIPLINARY SIMULATION OF COMPOSITE ENGINE STRUCTURES IN 


\title{
COUPLED MULTI-DISCIPLINARY SIMULATION OF COMPOSITE ENGINE STRUCTURES IN PROPULSION ENVIRONMENT
}

\author{
Christos C. Chamis \\ National Aeronautics and Space Administration \\ Lewis Research Center \\ Cleveland, Ohio 44135
}

and

\author{
Surendra N. Singhal \\ Sverdrup Technology, Inc. \\ Lewis Research Center Group \\ Brook Park, Ohio 44142
}

\section{ABSTRACT}

A computational simulation procedure is described for the coupled response of multi-layered multi-material composite engine structural components which are subjected to simultaneous multi-disciplinary thermal, structural, vibration, and acoustic loadings including the effect of hostile environments. The simulation is based on a 3D finite element analysis technique in conjunction with structural mechanics codes and with acoustic analysis methods. The composite material behavior is assessed at the various composite scales, i.e., the laminate/ply/constituents (fiber/matrix), via a nonlinear material characterization model. Sample cases exhibiting nonlinear geometrical, material, loading, and environmental behavior of aircraft engine fan blades, are presented. Results for deformed shape, vibration frequencies, mode shapes, and acoustic noise emitted from the fan blade, are discussed for their coupled effect in hot and humid environment. Results such as acoustic noise for coupled composite-mechanics/ heat transfer/structural/vibration/acoustic analyses demonstrate the effectiveness of coupled multi-disciplinary computational simulation and the various advantages of composite materials compared to metals.

\section{INTRODUCTION}

The unquenching thirst of the human race for better and newer technology keeps imposing bigger challenges. One of the key human endeavors successfully materialized was the ability to fly. The oncoming challenge is to fly faster than ever before. Fulfillment of this challenge is going to require new materials and new simulation approaches to designing aircrafts that can survive hostile environments at speeds which are multiples of the speed of sound. The new breed of materials, known as elevated temperature composites, which are light in weight, high in strength and durability, and can be tailored for required performance, are already finding applications and acceptance in aircraft frame and engine structures. There already are many competing requirements such as minimum temperature gradient versus maximum pressure tolerance as well as equally important considerations such as minimum noise. Designing with composite materials poses additional challenges in the areas of material selection, fabrication, durability, and failure mechanisms. Further complexities such as the influence of hostile environments, 
nonlinear material/structural behavior, and coupling between responses induced by various discipline-specific loads, require complex analysis methods. Clearly, coupled multi-disciplinary analysis methods capable of simulating the many discipline-specific loads and their interaction with each other, are needed. Additionally, newly evolving materials which may respond to the various multi-disciplinary loads and environments in an yet unknown manner, have no pre-existing data base. Since the acquisition of even a minimum necessary new data base may not be possible within the oncoming twenty first century's short design-cycle time limits and within the competitive cost constraints, computational simulation methods must be developed for the coupled multi-disciplinary analysis of engine structures.

Some recently evolving multi-disciplinary analysis codes were discussed in a recent article in the Mechanical Engineering magazine (ref. 1). The need for developing coupled multi-disciplinary analysis methods/ codes has been recognized for quite some time. For example, a multi-faceted research program aimed at simulating coupled multi-disciplinary behavior of aircraft engine/frame structures made of advanced composite materials, is described in reference 2. A significant amount of research conducted for simulating the characteristics/performance of elevated temperature composite materials/structures has been embedded in user-friendly computer codes. This effort continues to evolve and contains a multitude of discipline-specific as well as integrated codes covering a vast spectrum of consistent, compatible, and interactive analysis methods (ref. 3). More recently, a stand-alone multi-disciplinary simulation procedure/code was developed by integrating the 3D finite element analysis method with several single-discipline (thermal, acoustic - ref. 4) codes including those for integrated composite mechanics (ref. 5).

The objective of the present paper is to demonstrate the computational simulation of the coupled multi-disciplinary behavior of multi-layered multi-material elevated temperature aircraft engine composite structures under simultaneous thermal, structural, and acoustic loadings in propulsion environment, typified in Figure 1.

\section{BRIEF DESCRIPTION OF SIMULATION PROCEDURE}

A general-purpose procedure was developed to computationally simulate the coupled multi-disciplinary heat transfer, structural, vibration, and acoustic analysis of high temperature composite structures in propulsion environment. All the disciplines are coupled for nonlinear geometrical, material, loading, and environmental effects.

A schematics of the procedure is shown in Figure 2. First, the Model Definition module generates the finite element model of the structure geometry, composite configuration, boundary conditions, and loadings. The resident mesh generator is capable of generating solids of revolution such as cylinders, cones, and general double curved surfaces of up to 360 degree rotation, with minimum input parameters needed to define the complicated geometries. In case of combined different types of solids of revolution/flat surfaces, duplicate nodes are automatically checked and only one of each 
coincident nodes is kept. The Integrated Composite Analysis module, ICAN (ref. 5) is then used for determining the thermal and mechanical properties at various scales (fiber/matrix constituents, ply, and laminate) of the composite structure, based on composite micro-mechanics and laminate theories, starting from the room temperature properties of the constituents. A nonlinear material characterization model (ref. 6) shown in Figure 3, is used at the matrix level to simulate the degradation in material properties due to applied temperature, time, and environmental effects, etc. via an iterative approach, as shown on the left hand side of Figure 4. The ICAN module thus makes it possible to automatically compute the multi-scale composite properties of the virgin arbitrary combinations of multi-layered multi-material composite configurations, as well as for the degraded configurations at various stages of the composite structure life-cycle. The room temperature properties of fiber/matrix constituents for typical aircraft structure materials are automatically extracted from the ICAN resident data bank which can be augmented for properties of new materials. This feature results in a considerable saving of time required for searching and inputting the composite material property data. The ICAN module simulates the material behavior of polymer matrix composites. Similar codes (METCAN - ref.7, and CEMCAN - ref. 8) for simulating the material behavior of other types of elevated temperature composites such as metal matrix and ceramic matrix composites, respectively, are available. The simulation procedure can be adopted for these types of composites with minimal effort.

The computational procedure for coupled heat transfer, structural, vibration, and acoustic analysis is based on the 3D finite element formulation. Each element can be modelled as consisting of several layers of the composite material. Each layer can be arbitrarily oriented and be of different material. The heat transfer response is computed first via the Thermal Analysis module, THEAN. Four types of heat transfer analyses: (1) linear steady state, (2) nonlinear steady state, (3) linear transient, and (4) nonlinear transient, can be performed, with thermal properties computed and updated via the ICAN module. All types of thermal loadings including prescribed temperatures, surface heat fluxes, convection, radiation, and internal heat generation can be applied. Upon completion of the heat transfer analysis, the temperature at each node of the structure is defined.

The same finite element mesh that was used for the heat transfer analysis, is also used for the structural and vibration analyses. This minimizes the data preparation time and eliminates the errors incurred in transforming the temperatures from one finite element mesh to another. Two types of structural analyses: (1) static and (2) buckling can be performed, at the end of any heat transfer analysis step. All types of loadings including displacements, forces, accelerations, centrifugal, and pressure can be applied. The pressure can very across the element face. Several coordinate systems including global, skew, local, material, and for micro-mechanics, are used to allow maximum flexibility in inputting data at any orientations for complex structures. For example, skew coordinate system allows input of skew boundary conditions. The vibration analysis computes the free vibration frequencies and mode shapes. Free vibration frequencies 
and mode shapes can be calculated using the "determinant search" or the "subspace iteration" method. The effect of environment (temperature and moisture) on the structural and vibration response of the material is accounted for via the ICAN module. Nonlinear geometric effects such as large deformation/centrifugal stiffening are accounted for via updated Lagrange analysis. In such cases; (1) the pressure can be computed for either the original or the updated deformed geometry and (2) free vibration frequencies and mode shapes are calculated including the effect of updated geometry on the stiffness and mass matrices. Critical buckling loads also use updated stiffness for large deformation. The local structural response at ply and fiber/matrix scales of the composite structure, can be computed via ICAN, as shown on the right hand side of Figure 4.

The acoustic analysis module, ACOAN computes the acoustic noise emitted from the composite structure, due to (1) free vibration, or (2) forced vibration induced by applying a force at a point of the structure to selectively excite the vibration modes of interest. The acoustic noise is computed by first calculating radiation efficiencies of the structure for each natural vibration mode as a function of forced vibration frequency. The total sound power for each forced vibration frequency is then calculated by summing the contribution from each free vibration mode. The computation of acoustic noise includes (1) the effect of updated geometry due to large deformation via (a) updated structure stiffness and mass, and (b) updated geometry of the structure affecting the location and direction of the acoustic excitation force, (2) the effect of environment on thermal and mechanical properties via updated structure stiffness, and (3) the effect of natural vibration frequency on the modal loss factor (damping). A part of the structure can be masked from emitting the noise.

Finally, the multi-load step analysis feature allows multi-directional coupling among all the participating disciplines by passing the updated geometry and updated material behavior back to any one or all analysis disciplines via a nonlinear iterative procedure.

The coupling between the various disciplines due to geometrical, material, loading, and environmental complexities described above, allows many combinations of coupled multi-disciplinary analyses, as will be demonstrated in the next section for a fan blade. There are several other advanced features in this computational simulation procedure. They are not discussed here as they are beyond the scope of the demonstration cases presented in this paper. An example is the two-way coupling of the updated geometry due to large deformation and the heat transfer analysis via multi-load increment analysis capability.

\section{SAMPLE CASES - FAN BLADE}

A multi-material, multi-layered aircraft engine fan blade was simulated for a coupled multi-disciplinary thermal, structural, vibration, and acoustic response. The geometry, boundary conditions, material, composite configuration, environment, and 
boundary conditions, material, composite configuration, environment, and thermal/structural/ acoustic loadings are shown in Figure 5. A summary of the various analysis disciplines, and coupling effects demonstrated for the fan blade, is provided in Table 1. The design requirements may include other considerations such as aerodynamics of the blade. These are beyond the scope of this paper and have been and are being addressed via other integrated codes (ref. 3). A description of the loadings, boundary conditions, environmental effects, and of the single-discipline or coupled multi-discipline analyses performed for the fan blade follows the description of the blade structure, material, and the finite element model.

\section{Blade Structure, Material, and the Finite Element Model}

The blade consists of a twisted aerofoil shape with varying thickness along the span and the chord. The maximum span of the blade is 10.19 inches and the maximum chord is 3.43 inches. The thickness varies from 0.20 at the leading edge tip to 1.35 inches at the trailing edge tip. As noted in Figure 5, the blade is analyzed for two materials; (1) metal - titanium, and (2) multi-layered multi-material composite - $40 \%$ thickness of titanium and $60 \%$ thickness of six-layered T300/IMHS material with $(0 / 30 /-$ $30)_{s}$ ply orientations and 0.6 fiber volume ratio. The T300 stands for the graphite fibers and IMHS for intermediate high strength epoxy matrix. The thermal and mechanical properties of the T300 fibers and IMHS matrix at room temperature are shown in Table 2. The finite element model consisted of 4020 -noded ( 8 corner and 12 mid-side) brick elements with 110 nodes.

\section{Heat Transfer}

The root of the blade was held at a constant temperature of $200^{\circ} \mathrm{F}$ and the blade surface was subjected to fluid flow at $300^{\circ} \mathrm{F}$. The thermal material properties; thermal conductivity and coefficient of heat convection, were considered temperature-dependent via (a) direct input of temperature-dependent properties for the metal, and (b) ICAN for the composite. A nonlinear steady-state heat transfer analysis coupled with composite mechanics was conducted for both materials.

\section{Structural Analysis}

The root of the blade was fixed and a centrifugal loading of $1400 \mathrm{rpm}$ was applied. The effect of propulsion environments was simulated for several cases: (1) room temperature $\left(70^{\circ} \mathrm{F}\right)$ with no moisture absorption, (2) uniform temperature rise from 70 to $300{ }^{\circ} \mathrm{F}$ with no moisture absorption, (3) nonuniform temperature distribution as computed by the heat transfer analysis with no moisture absorption, and (4) nonuniform temperature distribution as computed by the heat transfer analysis with $2 \%$ absorption of moisture in the composite, by weight. Notice that cases (3) and (4) with nonuniform temperature distribution require coupled composite-mechanics/heat-transfer/structural analysis. A fifth case (5) was simulated for the effect of geometric stiffening at room temperature and no moisture. The thermal and mechanical properties; coefficient of thermal expansion, stiffness, and strength, were considered temperature-dependent via (a) direct input of temperature-dependent properties for the metal, and (2) ICAN for the 


\section{Vibration}

The root of the blade was fixed. Five vibration analysis cases similar to those described above for the "Structural Analysis", were simulated. Again, cases (3) and (4) with nonuniform temperature distribution computed via heat transfer analysis, require a coupled composite-mechanics/heat-transfer/vibration analysis. And, case (5) with geometricstiffening, requires acoupled composite-mechanics/structural/vibrationanalysis to account for the effect of updated geometry due to geometric stiffening. The thermal and mechanical properties were also treated similar to those described above for the "Structural Analysis". Three natural vibration frequencies and mode shapes were computed for all five cases for both materials.

\section{Acoustic Excitation}

The root of the blade was fixed and sinusoidal forced vibrations of $10 \mathrm{lb}$ amplitude were applied at the leading edge tip in the blade thickness direction, at several forcing frequencies ranging from 100 to $1000 \mathrm{cps}$. Again, five cases with thermo-mechanical properties as discussed above were simulated. Cases (1) and (2) with room temperature and uniform temperature distribution, respectively, require coupled compositemechanics/vibration/acoustic analysis. Cases (3) and (4) with nonuniform temperature distribution computed via heat transfer analysis, require coupled compositemechanics/heat-transfer/vibration/acoustic analysis, and case (5) with geometric stiffening, requiresa coupled composite-mechanics/structural/vibration/acoustic analysis. A special variation of case (3) with frequency-dependent modal loss damping factor was also run. This is just one of many special features of the computational simulation procedure demonstrated in the present paper. The modal loss factors used for this evaluation are only estimates. The computational simulation of damping in composite materials is described in reference 9 .

\section{RESULTS AND DISCUSSIONS}

The results of several cases of the fan blade with varying degree of complexity/coupling among various disciplines, are discussed below in the same order in which the sample cases are described above. The effect of coupling among different disciplines is explained, as appropriate.

\section{Heat Transfer}

The heat transfer results in terms of temperature contours for both, the metal and composite blades, are shown in Figure 6. Notice that the computed temperatures vary from 200 to $285^{\circ} \mathrm{F}$ for the metal and from 200 to $275^{\circ} \mathrm{F}$ for the composite blade.

\section{Structural Analysis}

Only a representative result, i.e., the deformed shape of the blade for the case with nonuniform temperature distribution computed via heat transfer analysis and with no moisture absorption and no effect of geometric stiffening, is shown in Figure 7 for both, the metal and composite blades. The metal blade shows more radial deformation than 
the composite blade. The blade deforms less at the room temperature and more at 300 ${ }^{\circ} \mathrm{F}$ uniform temperature. The geometric stiffening reduces the blade deformation. These results are not shown in the Figure form in the present paper. The results for global and local stresses at ply and fiber/ matrix constituents scales are also available, but not presented here.

\section{Vibration}

The first three natural vibration frequencies for the case with nonuniform temperature distribution computed via heat transfer analysis and with no moisture absorption and no geometric stiffening, are shown in Figure 8. The corresponding mode shapes are shown in Figure 9. The effects of various environmental conditions (temperature/moisture) and geometric stiffening on the fundamental vibration frequency, are shown in Figure 10. The vibration frequency decreases with increasing temperature and moisture as the material degrades. The moisture does not affect the vibration frequency of the metallic blade. Geometric stiffening increases the vibration frequency by about 6 percent in the metal and about 5 percent in the composite as would be expected from the heavier metal density.

\section{Acoustic Excitation}

The acoustic noise emitted from the fan blade, is shown in Figure 11, in watts as well as in decibels. To put the decibel values in perspective, the human whisper is at 50 decibels, a truck horn is at 110 decibels, and an airplane engine propeller is at 120 decibels. Increasing damping decreases the noise level. The noise is much less for the composite material than for metal. Figure 12 shows the acoustic noise for various cases of environmental conditions and geometric stiffening. The acoustic noise decreases or increases depending on whether the forcing frequency is farther away from or closer to the natural vibration frequency, respectively. The effect of environment on the acoustic noise from the metallic fan blade is negligible. At temperatures higher than those used here, it may not be so. For the composite blade, increasing temperature and moisture increase the acoustic noise at the forcing frequency of $119 \mathrm{cps}$. These results are consistent with the results for the natural vibration frequencies shown in Figure 10, which become closer to $119 \mathrm{cps}$ as temperature and moisture increase. The effect of geometric stiffening is to decrease the acoustic noise because the forcing frequency moves away from the natural vibration frequency. Another effect, that of frequency dependence of the modal loss factors, is shown in Figure 13 for both, the metal and composite blades. The modal loss factor decreases with the vibration frequency, as tabulated in Figure 13. The acoustic noise increases, only slightly, by accounting for frequency dependence of the modal loss factors both, for the metal and composite blades.

The significant observation is that the acoustic noise from the composite fan blade is negligible compared to that from the metal blade, Figure 11. The effect of frequency dependence of the modal loss factors is practically the same for the two blades, Figure 13. 


\section{GENERAL REMARKS}

A stand-alone computational simulation procedure has been demonstrated for coupled response of an aircraft engine fan blade under multi-disciplinary thermal/structural/vibration/acoustic loading in propulsion environments. The procedure was developed via integrating the 3D finite element technique with in-house singlediscipline codes coupled with acoustic analysis methods. The simulation procedure is of general purpose in nature and can be used for designing/ analyzing multi-layered multimaterial composite structures. The results, such as the decrease in acoustic noise levels by accounting for structural/ acoustic coupling due to geometric stiffening, demonstrate the significance of coupled multi-disciplinary simulation of the blade. Results such as these can be generated via a single coupled multi-disciplinary code in a very short time. In an effort to satisfy the competing requirements imposed by individual discipline-specific behavior, many design variations/ parameters will need to be considered. Multidisciplinary computational simulation is the approach that will provide a realistic assessment of the various competing design requirements of advanced composite materials/structures. Unlike experimental data generation that may be untimely and costly, computational simulation is able to produce rapid reasonable results for specific designs.

The environmental effects discussed herein include temperature and moisture. Other environmental effects such as chemical interactions can be incorporated in the same way. The effect of chemical interaction on the degradation of material resistance can be simulated via the Integrated Composite Analyzer Code, ICAN, by adding respective terms in the nonlinear material characterization model, shown in Figure 3 (ref. 6). The modified form of the nonlinear material characterization model will consist of a product of nonlinear factors for each environmental effect. Such nonlinear multi-factor interaction models (MFIM) for factor such as cyclic effect have been implemented in metal matrix composite analysis codes (ref. 7). The effect of chemical interaction on changes in the geometry, if any, can be addressed in the finite element models by suitable changes in the dimensions. These effects can then be passed on to the multi-disciplinary analysis via the geometry update module.

\section{SUMMARY}

A general-purpose computational simulation procedure is presented for coupled multi-disciplinary thermal, structural, vibration, and acoustic analysis of elevated temperature composite structures in propulsion environments. All the disciplines are coupled for nonlinear geometrical, material, loading, and environmental effects. The procedure is embedded in a stand-alone state-of-the-art computer code, enabled by integrating the 3D finite element technique with in-house codes for integrated composite mechanics, thermal, and acoustic analysis methods. A nonlinear material characterization model is used to simulate the degradation in material properties due to applied 
temperature, time, and environmental effects. Sample cases exhibiting various combinations of coupled multi-disciplinary analysis of an aircraft engine fan blade, are presented. Results indicate lower temperatures, higher vibration frequencies, and substantially lower acoustic noise levels for the T300/IMHS composite versus that for titanium.

\section{REFERENCES}

1. Puttre, M., "FEA Programs Band Together", Mechanical Engineering Magazine, Vol, 113, No. 9, pp. 77-82, Sept., 1991.

2. Chamis, C.C., "Computer Code for the Analysis of Multi-Layered Fiber Composite User's Manual", NASA TN - D - 7013, 1971.

3. "A Guide to NASA Lewis Structural Mechanics Codes", NASA Report (under preparation), 1991.

4. Hayes, P.E., "Determining Vibration, Radiation Efficiency, and Noise Characteristics of Structural Designs Using Analytical Techniques", Proceedings - Society of Automotive Engineers, p-106, SAE, Warrendale, PA, pp. 236-270, 1982.

5. Murthy, P.L.N. and Chamis, C.C., "Integrated Composite Analyzer (ICAN) - User's and Programmer's Manual, NASA TP 2515, March, 1986.

6. Chamis, C.C., "Simplified Composite Micro-Mechanics Equations for Hygral, Thermal, and Mechanical Properties", NASA TM-83320, 1987.

7. Lee, H. J., Gotsis, P. K., Murthy, P.L.N., and Hopkins, D. A., "Metal Matrix Composites Analyzer: METCAN User's Guide", NASA Report, 1991.

8. Murthy, P.L.N. et.al., "Ceramic Matrix Composite Analyzer: CEMCAN", NASA Report (under preparations), 1991.

9. Saravanos, D.A. and Chamis, C.C., "Mechanics of Damping for Fiber Composite Laminates Including Hygrothermal Effects", AIAA Journal Vol, 28, \#10, $p p 1813$ $1819,1990$. 


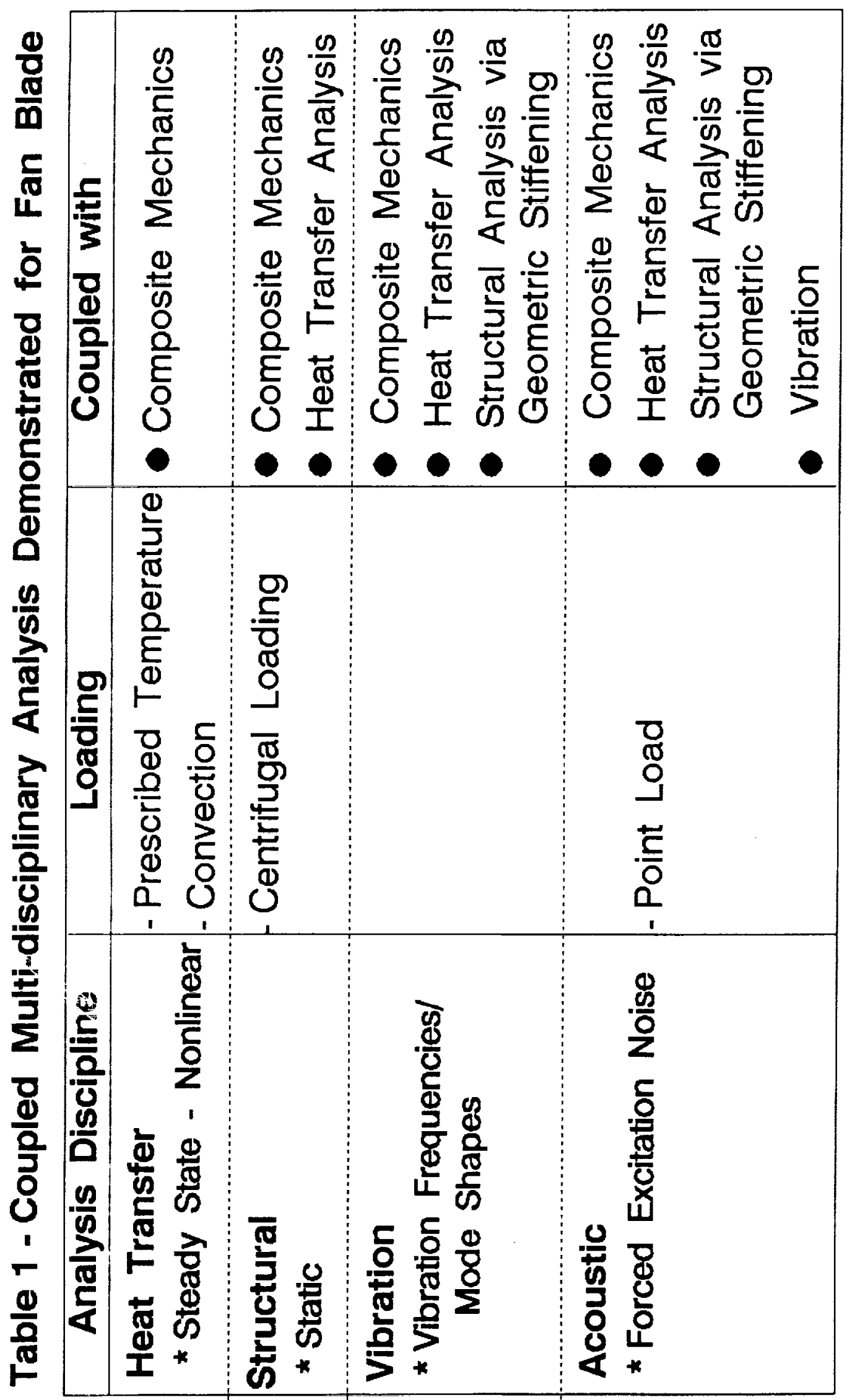




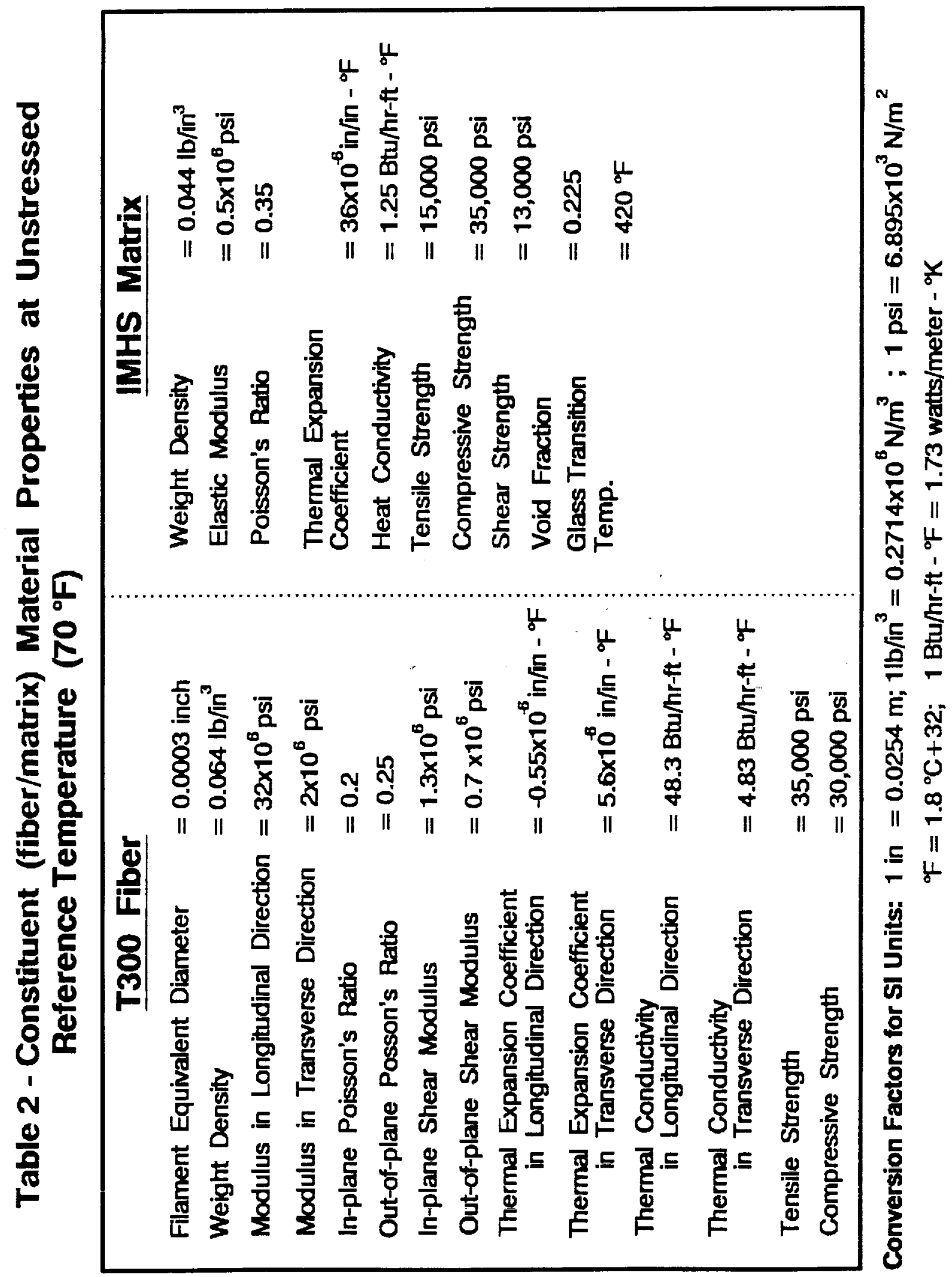




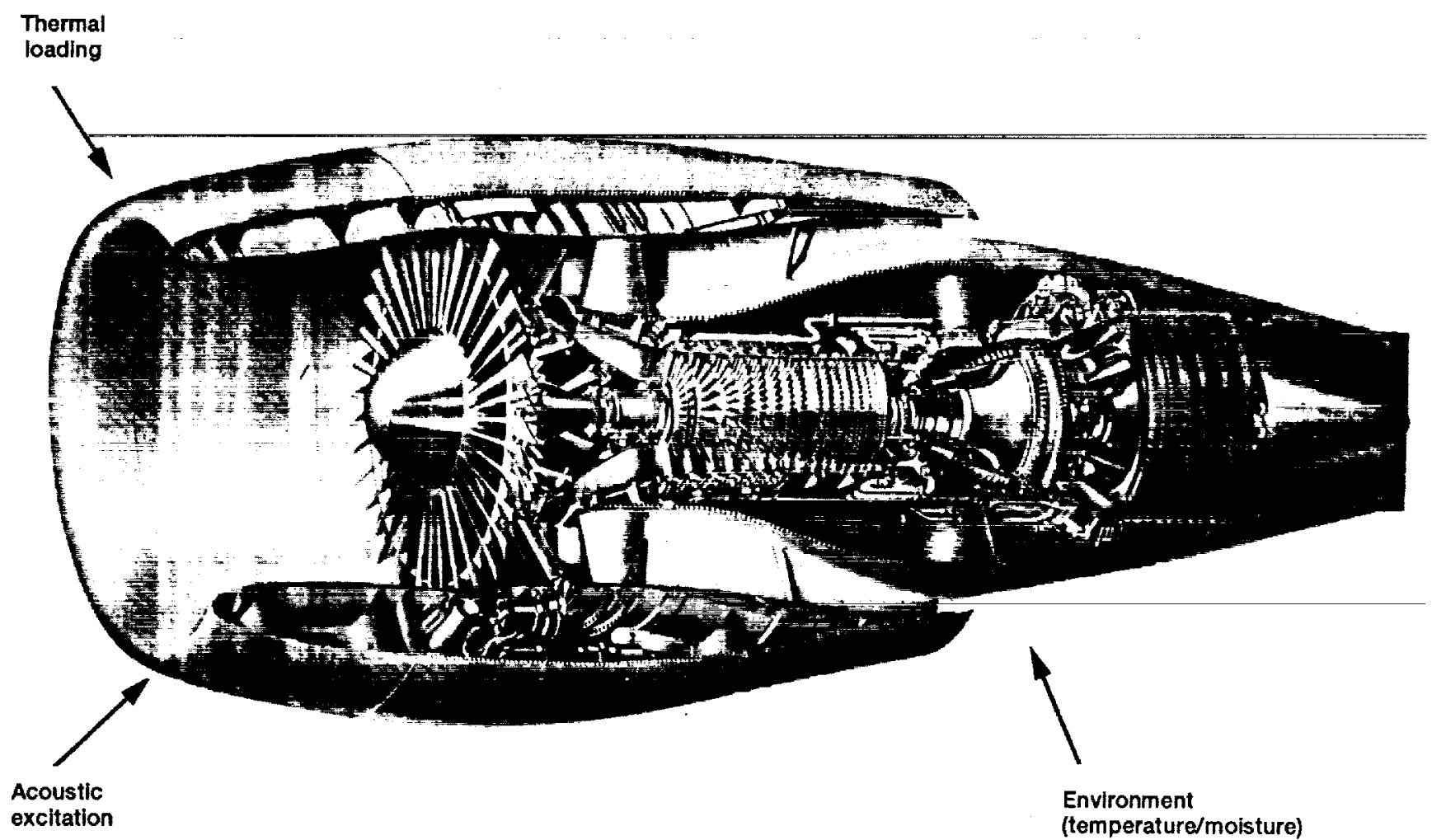

Figure 1.-Engine components under multidisciplinary loadings. 


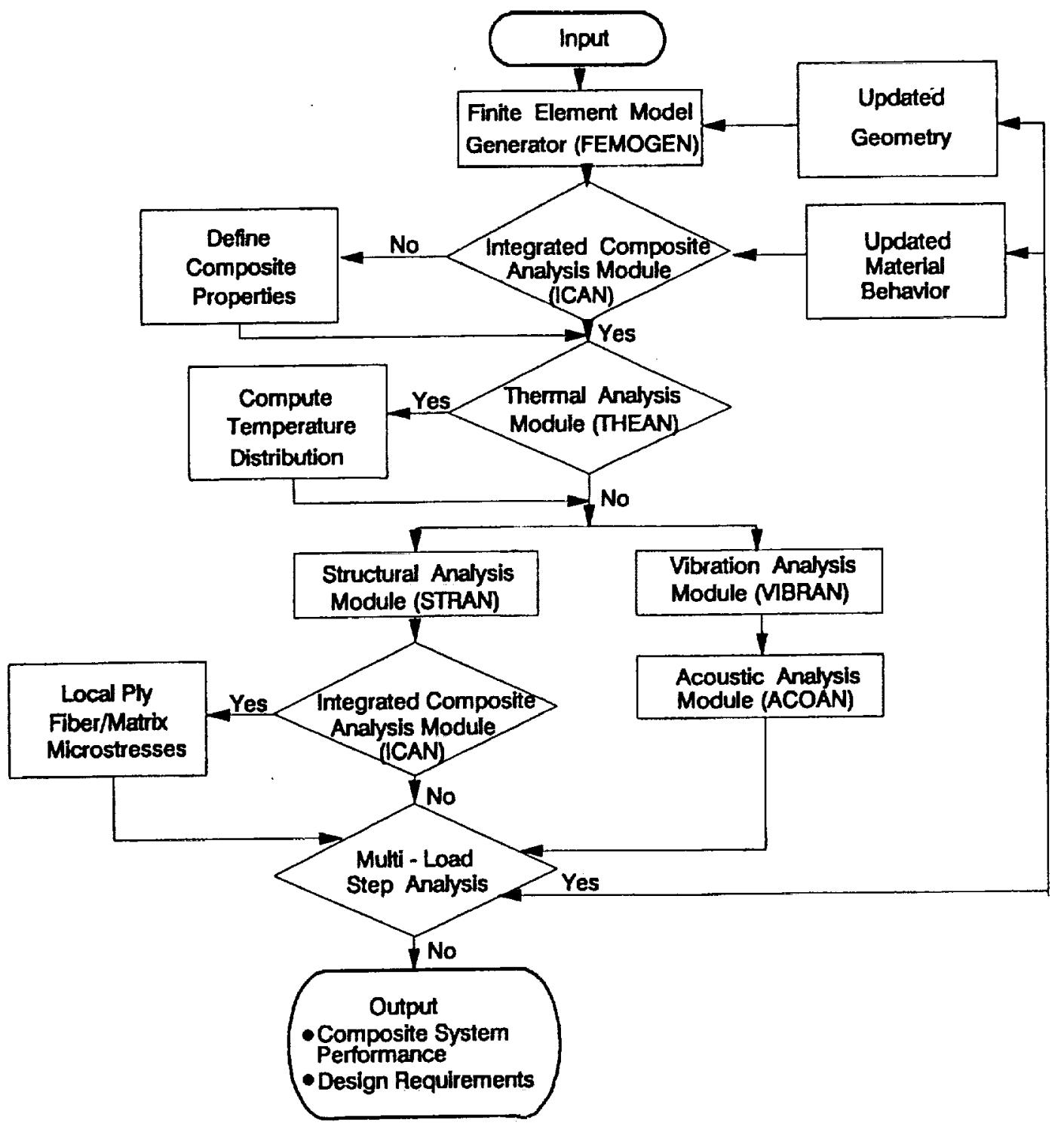

Flgure 2.-Multt-dlsciplinary simulation procedure. 


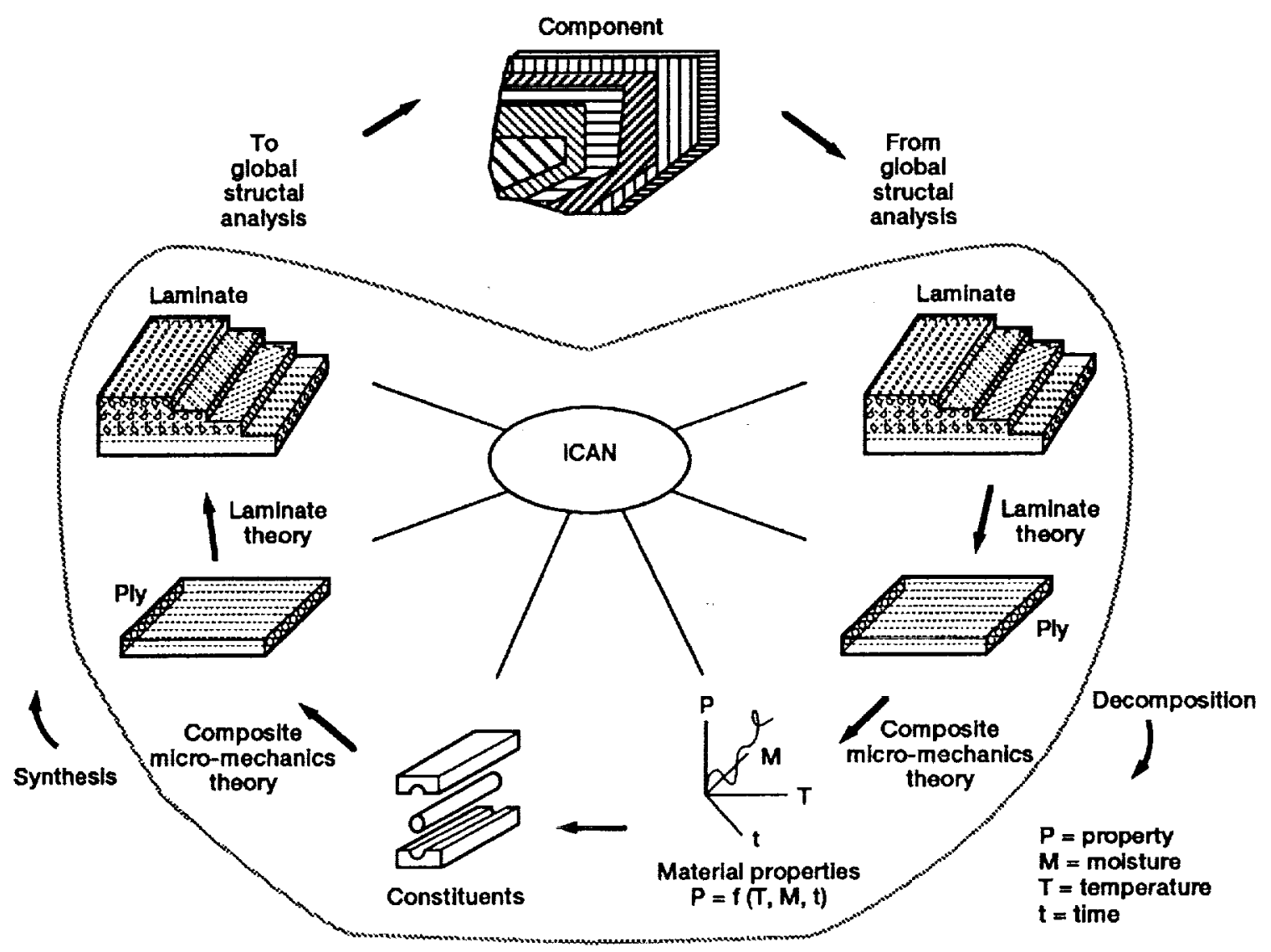

Figure 4.- Integrated composite mechanics - ICAN.

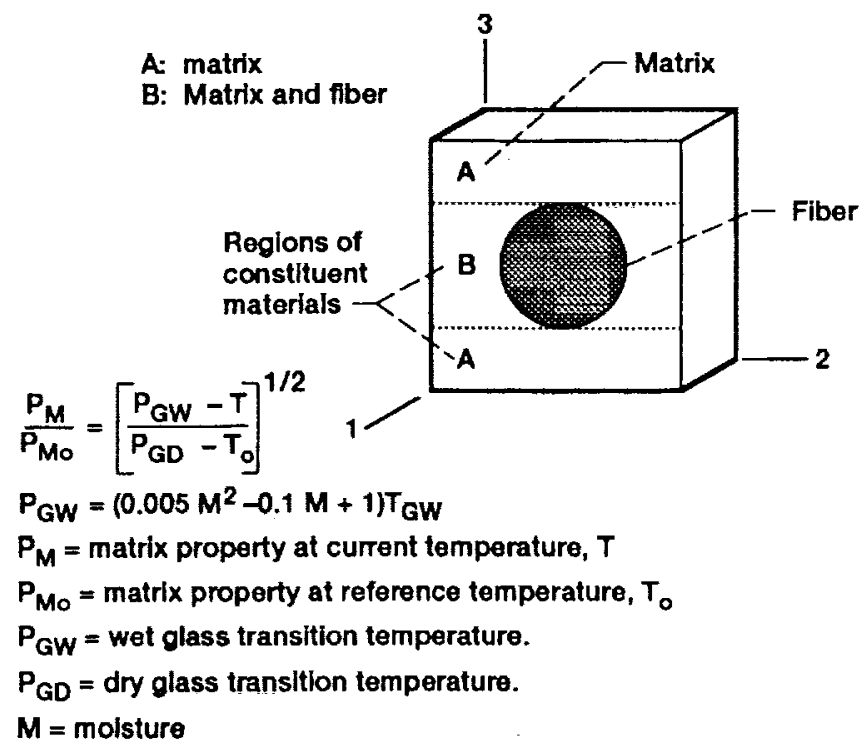

Flgure 3.-Reglons of constltuent materials and nonlinear material characterization model. 
Acoustic loading (10 lb in $\mathrm{z}$ direction) Forcing Function $(F)$ vs. Frequency $(\omega)$

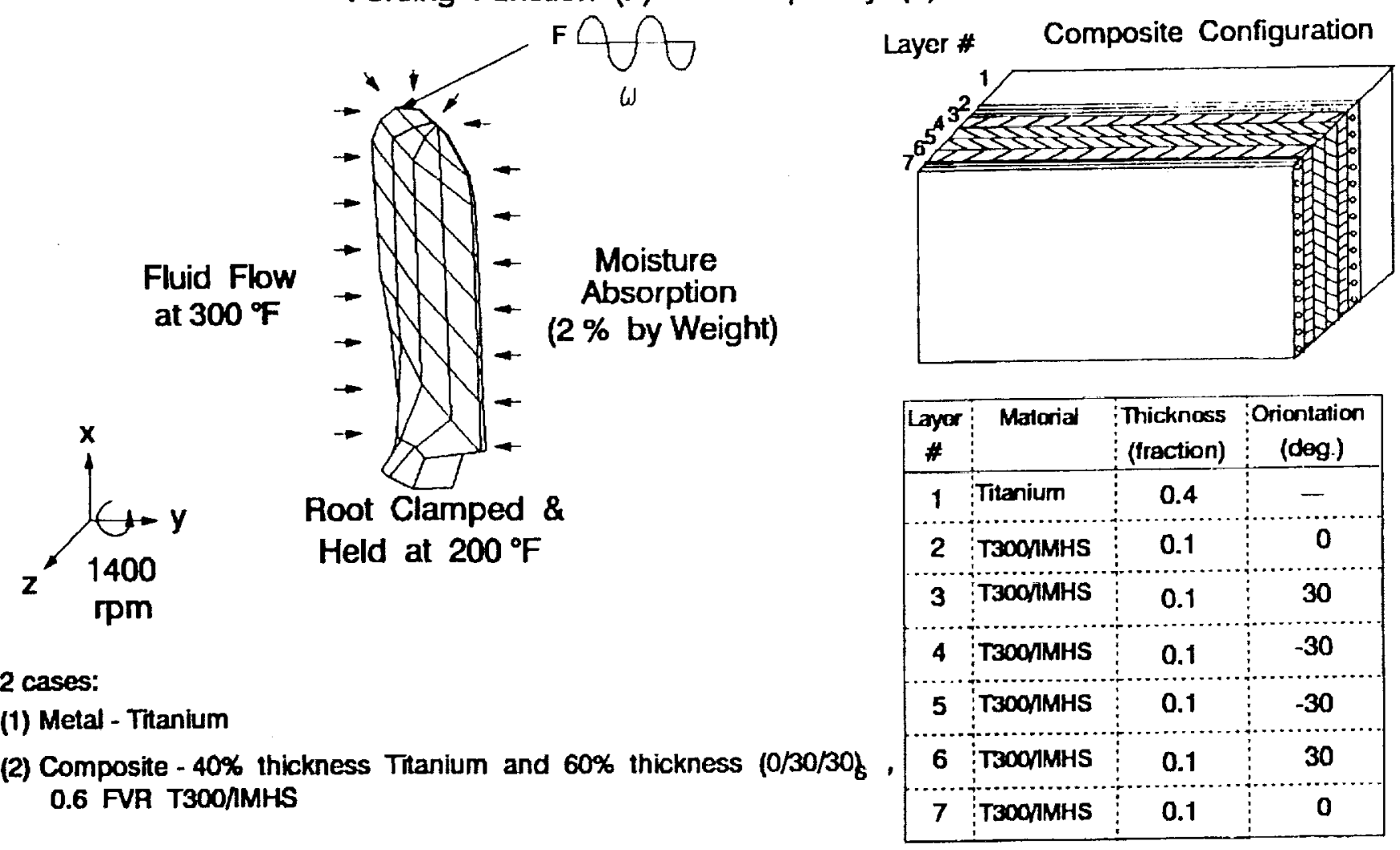

Flgure 5.-Fan blade under multi-disciplinary loading.

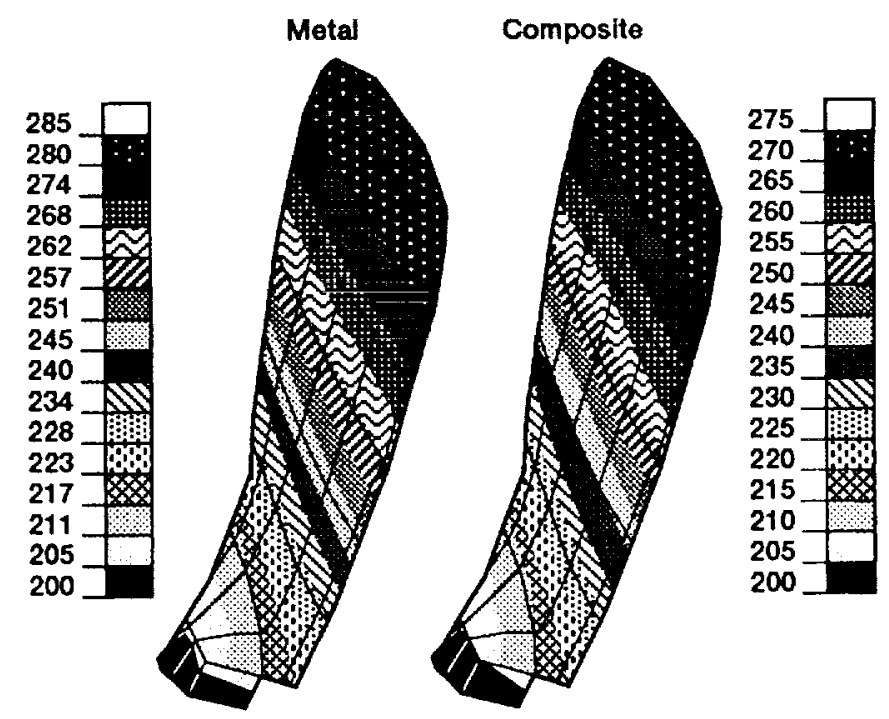

Figure 6.-Thermal response of fan blade. Temperature ${ }^{\circ} \mathrm{F}$ contours via coupled composite-mechanics/heat-transfer analysis. (Root $\odot 200^{\circ} \mathrm{F}$; fluld flow $300^{\circ} \mathrm{F}$ ) 
Deformed Shape via Coupled Composite-Mechanics/Heat-Transfer/Structural Analysis (Centrifugal Loading at 1400 rpm; Root @ 200 \%F; Fluid Flow @ 300 \%)

\section{Metal}

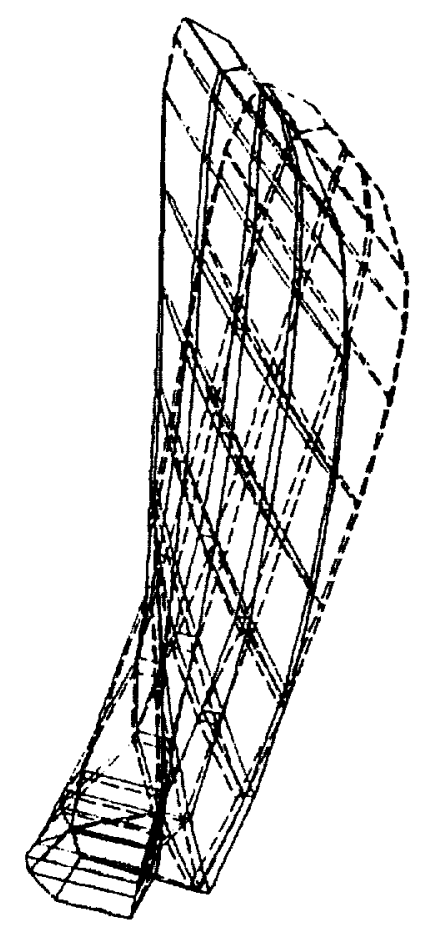

\section{Composite}

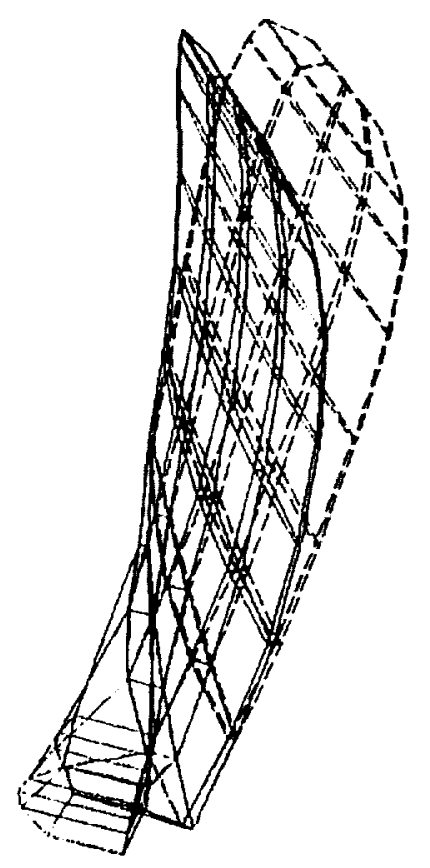

Flgure 7.- Structural response of fan blade. 
Vibration Frequencies via Coupled Composite-Mechanics/Heat-Transfer/Vibration Analysis (Root @ 200 F; Fluid Flow @ 300 F)

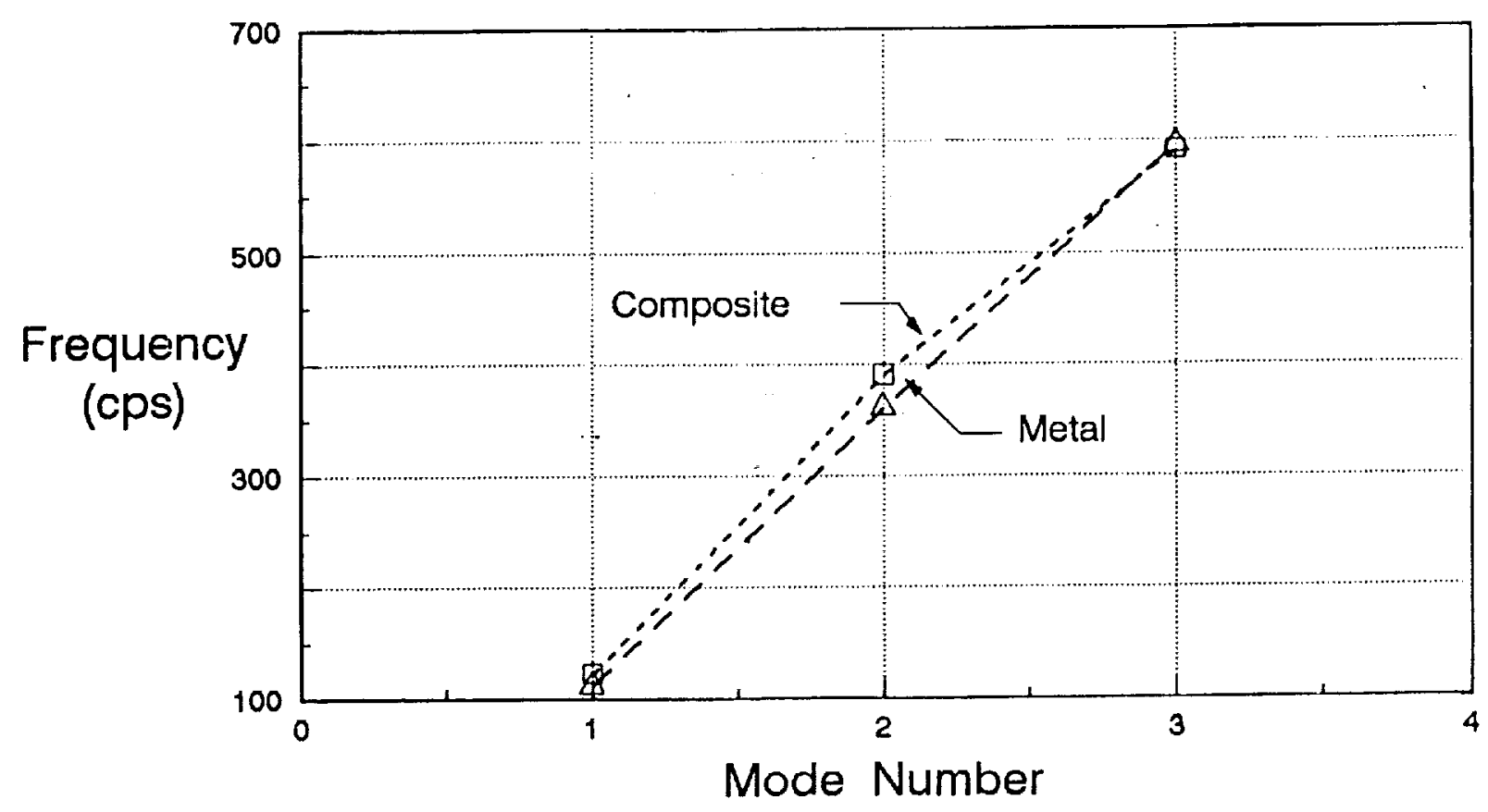

Figure 8.-Vibration response of fan blade - frequencles. 
Vibration Modes via Coupled Composite-Mechanics/Heat-TransferNibration Analysis (Root@ @ $200^{\circ} \mathrm{F}$; Fuid Flow @ $300^{\circ} \mathrm{F}$ )

\section{Metal}
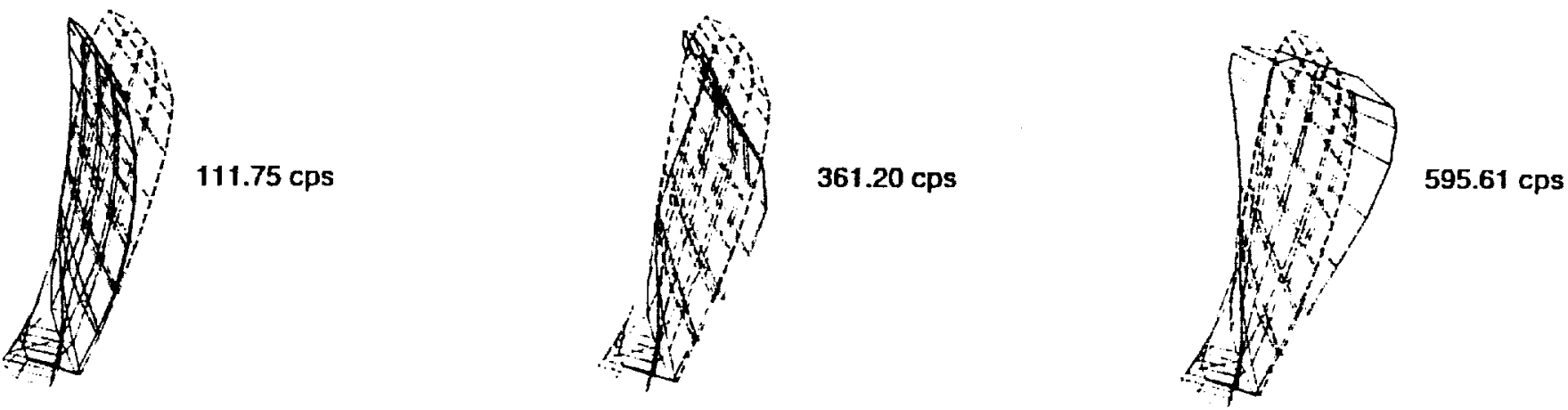

\section{Composite}
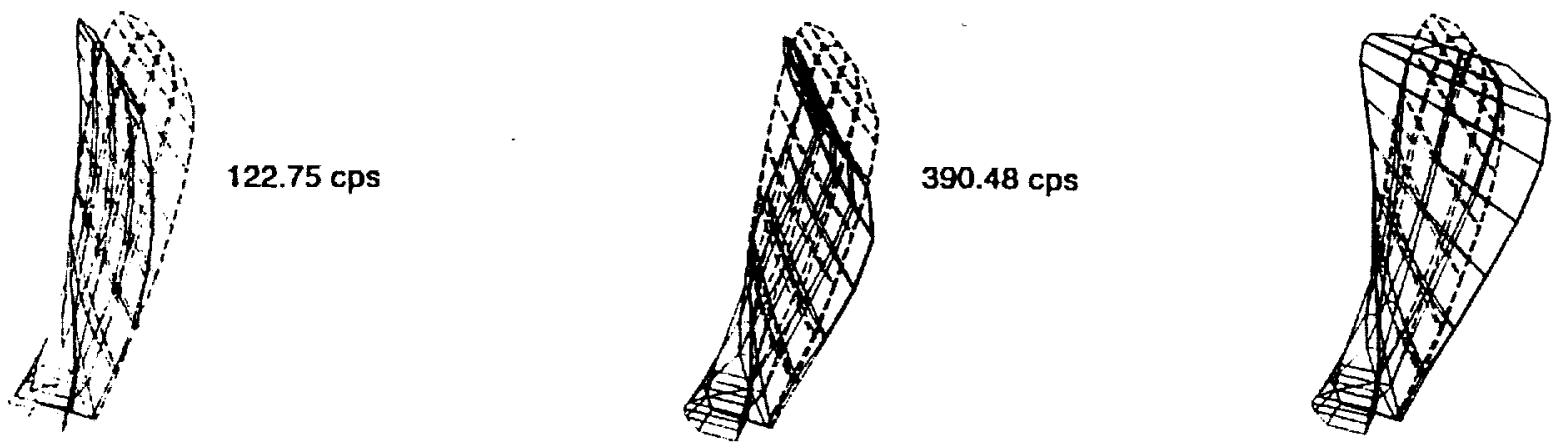

591.34 cps

Figure 9.-Vlbration response of fan blade - mode shapes. 
Vibration Frequencles via Coupled Composite-Mechanics/Heat-Transfer/Structura/Nibration Analysis Effect of Environment (Temp./Moisture) and Geometric Stiffening on Fundamental Frequency

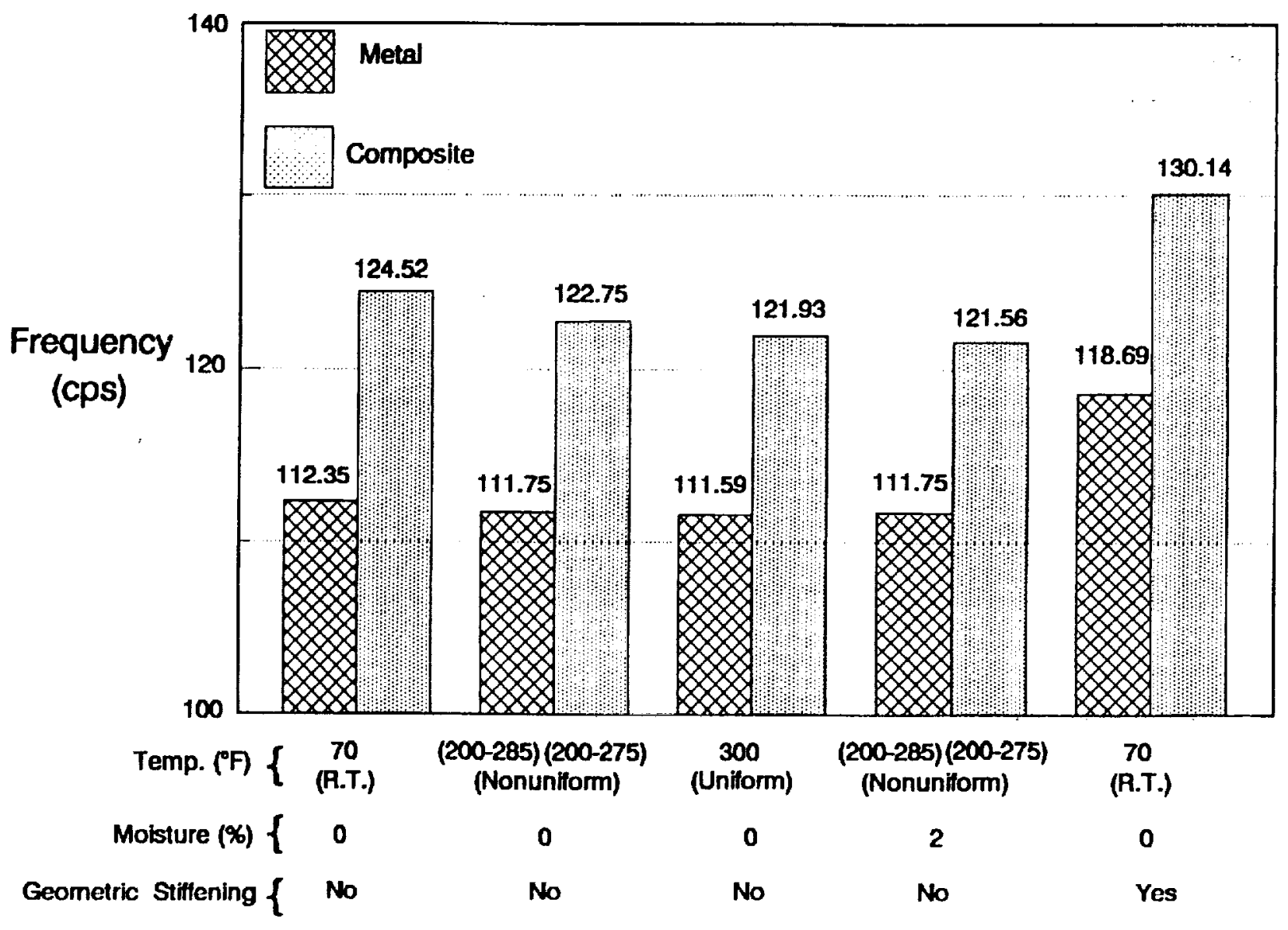

Figure 10.-Vibration response of fan blade - fundamental frequency. 
Acoustic Noise via Coupled Composite-Mechanics/Heat-Transfer/Nibration/Acoustic Analysis (Acoustic Excitation of $10 \mathrm{lb} @$ @ Leading Edge Tip; Rool Fixed @ $200^{\circ} \mathrm{F}$; Fluid Flow @ $300^{\circ} \mathrm{F}$ )

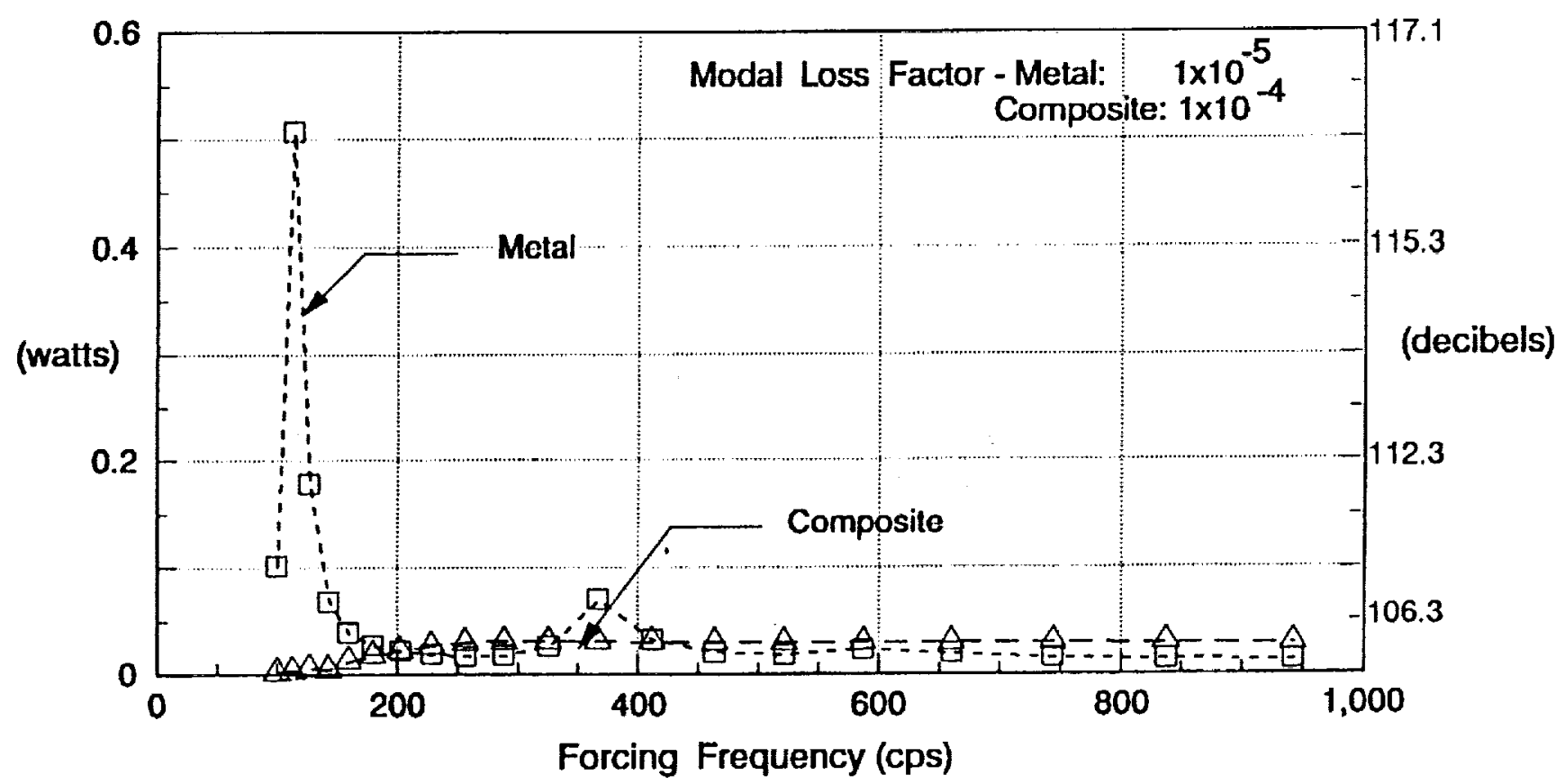

Figure 11. Acoustic nolse emitted from fan blade. 
Acoustic Noise via Coupled Composite-Mechanics/Heat-Transfer/Structural/ Vibration/Acoustic Analysis

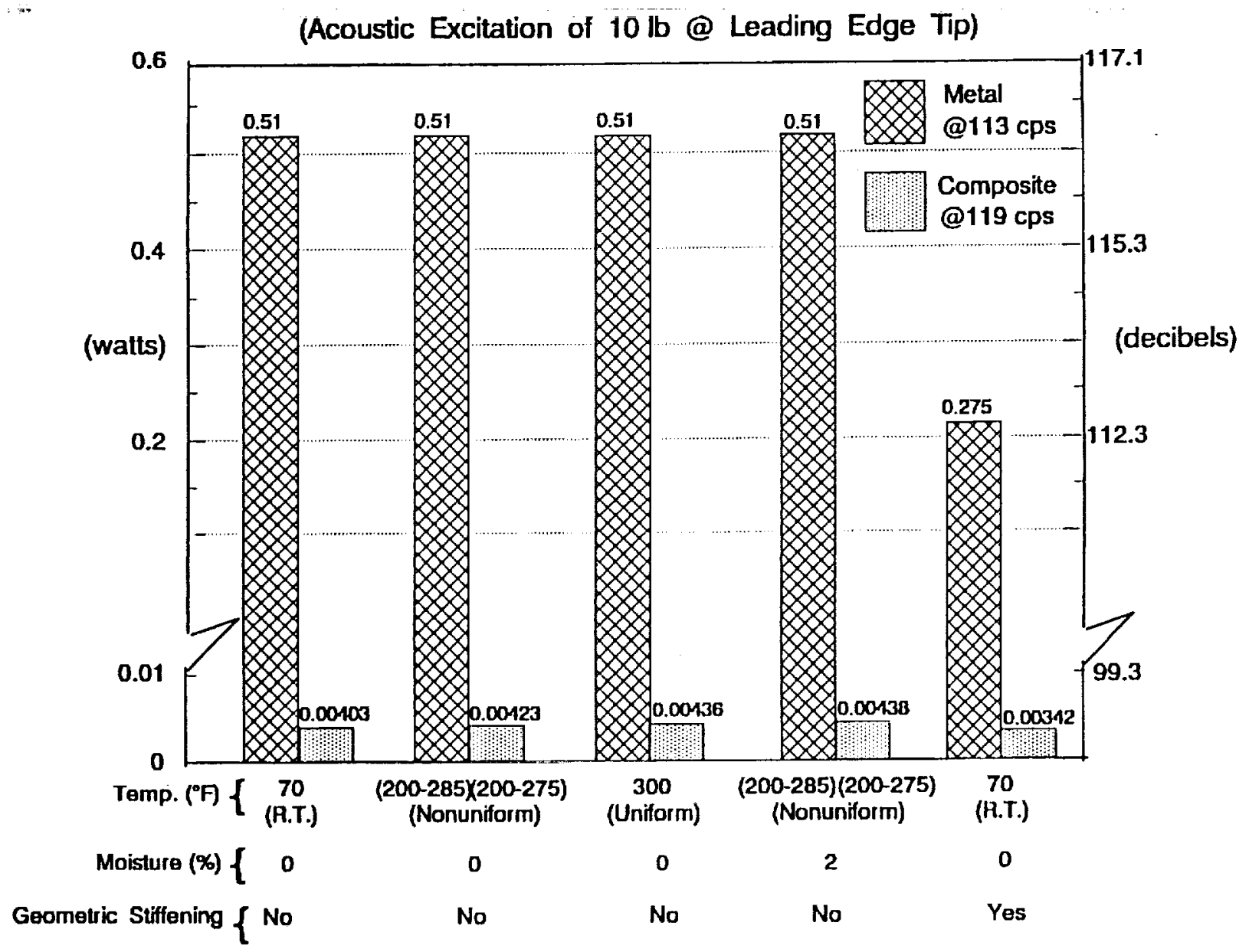

Figure 12.-Acoustic nolse emitted from fan blade - at specified forcing frequency. 
Acoustic Noise via Coupled Composite-Mechanics/Heat-Transfer/Nibration/AcousticAnalysis (Acoustic Excitalion of $10 \mathrm{lb} @$ @ Leading Edge Tip; Root Fixed @ $200^{\circ} \mathrm{F}$; Fluid Flow @ $300^{\circ} \mathrm{F}$ )
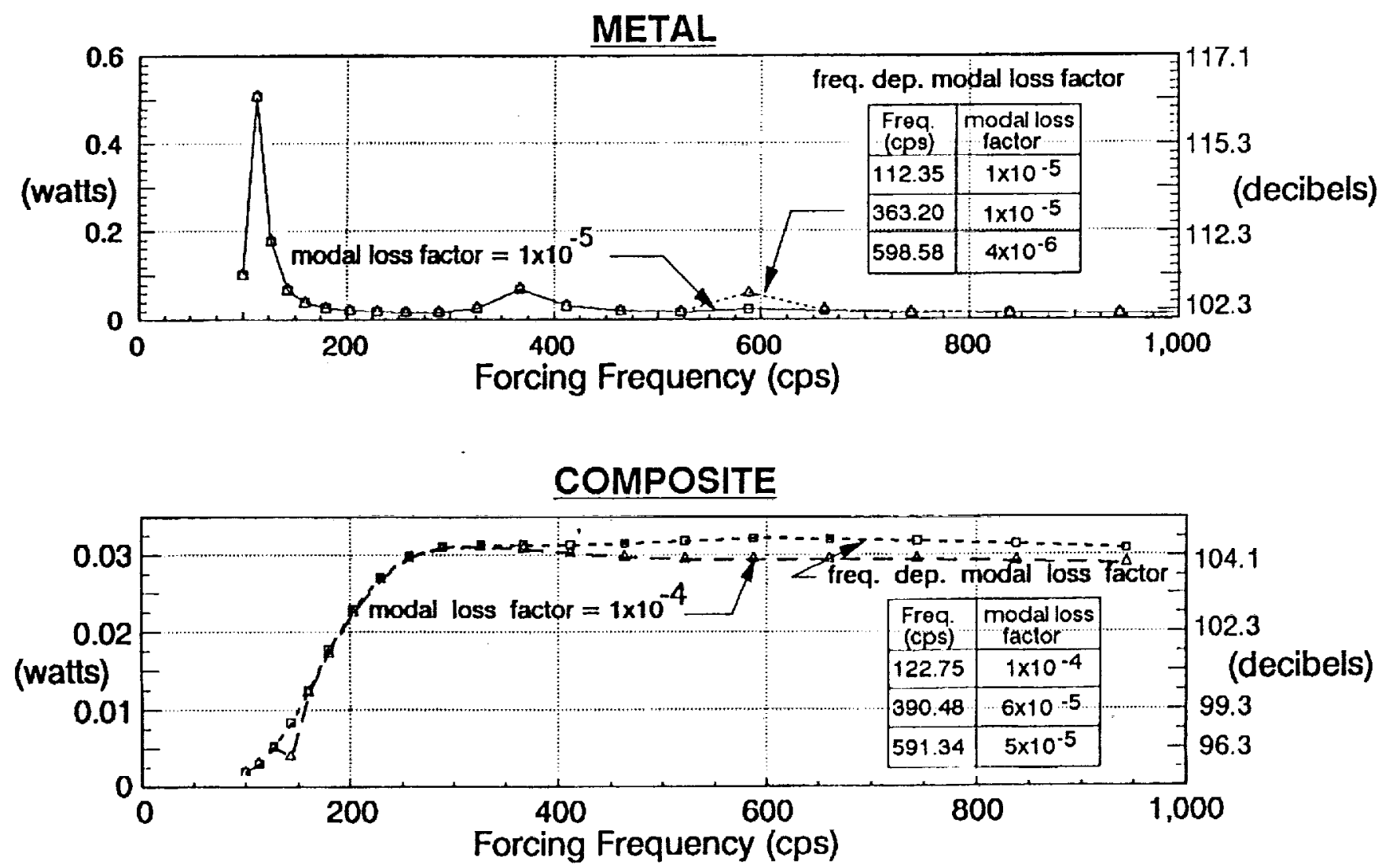

Figure 13.-Acoustic nolse emitted from fan blade - frequency dependent modal loss factor. 
、

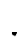


Public reporting burden lor this collection of information is estimated to average 1 hour per response, including the time for reviewing instructions, searching existing data sources, gathering and maintaining the data needed, and completing and reviewing the collection of information. Send comments regarding this burden estimate or any other aspect of this collection of information, including suggestions for reducing this burden, to Washington Headquarters Services, Directorate for information Operations and Reports, 1215 Jefferson Davis Highway, Suite 1204, Arlington, VA 22202-4302, and to the Office of Management and Budget, Paperwork Reduction Project (0704-0188), Washington, DC 20503.

\begin{tabular}{|l|c|c|}
\hline 1. AGENCY USE ONLY (Leave blank) & $\begin{array}{c}\text { 2. REPORT DATE } \\
\text { June } 1992\end{array}$ & $\begin{array}{c}\text { 3. REPORT TYPE AND DATES COVERED } \\
\text { Technical Memorandum }\end{array}$ \\
\hline
\end{tabular}

4. TITLE AND SUBTITLE

Coupled Multi-Disciplinary Simulation of Composite Engine Structures in Propulsion Environment

6. AUTHOR(S)

Christos C. Chamis, Surendra N. Singhal

\section{PERFORMING ORGANIZATION NAME(S) AND ADDRESS(ES)}

National Aeronautics and Space Administration

Lewis Research Center

Cleveland, Ohio 44135-3191

National Aeronautics and Space Administration

Washington, D.C. 20546-0001
5. FUNDING NUMBERS

WU-505-63-53

8. PERFORMING ORGANIZATION REPORT NUMBER

E-6901

10. SPONSORING/MONITORING AGENCY REPORT NUMBER

NASA TM - 105575

\section{SUPPLEMENTARY NOTES}

Prepared for the International Gas Turbine and Aeroengine Congress and Exposition, Cologne, Germany, June 1-4, 1992. Christos C. Chamis, NASA Lewis Research Center and Surendra N. Singhal, Sverdrup Technology, Inc., Lewis Research Center Group, 2001 Aerospace Parkway, Brook Park, Ohio 44142 (work funded by NASA Contract NAS3-25266). Responsible person, Christos C. Chamis, (216) 433-3252.

12a. DISTRIBUTION/AVAILABILITY STATEMENT 12b. DISTRIBUTION CODE

Unclassified - Unlimited

Subject Category 39

13. ABSTRACT (Max/mum 200 words)

A computational simulation procedure is described for the coupled response of multi-layered multi-material composite engine structural components which are subjected to simultaneous multi-disciplinary thermal, structural, vibration, and acoustic loadings including the effect of hostile environments. The simulation is based on a $3 \mathrm{D}$ finite element analysis technique in conjunction with structural mechanics codes and with acoustic analysis methods. The composite material behavior is assessed at the various composite scales, i.e., the laminate/ply/constituents (fiber/ matrix), via a nonlinear material characterization model. Sample cases exhibiting nonlinear geometrical, material, loading, and environmental behavior of aircraft engine fan blades, are presented. Results for deformed shape, vibration frequencies, mode shapes, and acoustic noise emitted from the fan blade, are discussed for their coupled effect in hot and humid environment. Results such as acoustic noise for coupled composite-mechanics/heat transfer/ structural/vibration/acoustic analyses demonstrate the effectiveness of coupled multi-disciplinary computational simulation and the various advantages of composite materials compared to metals.

14. SUBJECT TERMS

Acoustic excitation; Acoustic noise; Composites; Coupled analysis; Environment; Frequencies; Geometric stiffening; Heat transfer; Hygro-thermo-mechanics; Large deformation; Modal response; Moisture; Multi-disciplinary analysis; Polymer matrix composites; Simulation; Structural analysis; Temperature; Thermal response; Vibration

15. NUMBER OF PAGES 24

16. PRICE CODE

$\mathrm{A} 03$

\begin{tabular}{|c|c|c|}
\hline $\begin{array}{c}\text { 17. SECURITY CLASSIFICATION } \\
\text { OF REPORT } \\
\text { Unclassifjed }\end{array}$ & $\begin{array}{c}\text { 18. SECURITY CLASSIFICATION } \\
\text { OF THIS PAGE } \\
\text { Unclassified }\end{array}$ & $\begin{array}{c}\text { 19. SECURITY CLASSIFICATION } \\
\text { OF ABSTRACT } \\
\text { Unclassified }\end{array}$ \\
\hline
\end{tabular}

NSN 7540-01-280-5500
Standard Form 298 (Rev. 2-89)

Prescribed by ANSI Std. Z39-18 298-102 